\title{
Alltagspraktiken digitaler Teilhabe in Nachbarschaft und Stadt
}

\author{
Paula Nitschke / Moritz Schweiger*
}

Der Artikel untersucht Alltagspraktiken digitaler Teilhabe auf Ebene der Nachbarschaft und der Stadt anhand von Online-Nachbarschaftsplattformen. In theoretischer Hinsicht wird die Frage aufgeworfen, inwiefern Alltagspraktiken digitaler Teilhabe als mediale Integrationsleistungen zu verstehen sind, und es ist das Ziel des Artikels, das integrative Potenzial der Nachbarschaftsplattformen einzuschätzen. Dafür wird das Konzept Alltagspraktiken digitaler Teilhabe, bestehend aus den Dimensionen politische Partizipation und Vergemeinschaftung, aus der kommunikationswissenschaftlichen und stadtsoziologischen Literatur abgeleitet. Empirisch geprüft wurde das Konzept durch einen Methodenmix, bestehend aus standardisierter Inhaltsanalyse und qualitativen Interviews. Im Ergebnis zeigt sich, dass die Plattformen vor allem Vergemeinschaftung fördern. Auf der Plattform werden lose Beziebungen gepflegt und es werden enge Beziehungen zu Menschen mit gemeinsamen Interessen und Bedürnissen vermittelt. Das Zugehörigkeitsgefübl zur Nachbarschaft kann auch durch das alleinige Beobachten von Vergemeinschaftungspraktiken Anderer entsteben. Politische Partizipation ist auf den Plattformen hingegen weniger verbreitet und wird von den Nutzer*innen zudem ambivalent bewertet.

Schlüsselwörter: Alltagspraktiken, Digitale Teilhabe, Integration, Nachbarschaft, Partizipation, Stadt, Vergemeinschaftung

\section{The Everyday Practice of Digital Participation in Neighbourhoods and Cities}

The article examines everyday practices of digital participation in the neighbourbood and the city through an analysis of online neighbourhood platforms. From a theoretical point of view, the question is raised as to the extent to which everyday practices of digital participation perform an integrative function, and it is the aim of this article to assess the integrative potential of neighbourhood platforms. For these aims, the concept of everyday practices of digital participation, consisting of dimensions including community building and political participation is derived from of the fields of communication and media studies as well as urban sociology. The concept was empirically tested using a mixedmethods approach using standardised content analysis and qualitative interviews. Findings show that these platforms primarily promote community building. Loose relationships are cultivated on the platform and close relationships with people with common interests and needs are mediated. Moreover, a sense of belonging to the neighbourbood can arise from simply observing the community building practices of others. Political participation, on the other hand, is less common on the platforms and is also evaluated ambivalently by users.

Keywords: everyday practices, digital participation, community building, integration, participation, neighbourhood, city

* Dr. Paula Nitschke, Universität Augsburg; Institut für Medien, Wissen und Kommunikation (Arbeitsbereich Medienrealität), Universitätsstraße 2, 86159 Augsburg, paula.nitschke@ phil.uni-augsburg.de. ORCID: 0000-0001-7247-617X.

Moritz Schweiger, M. A., Universität Augsburg; Institut für Medien, Wissen und Kommunikation (Arbeitsbereich Medienrealität), Universitätsstraße 2, 86159 Augsburg, moritz.schwei ger@phil.uni-augsburg.de, ORCID: https://orcid.org/0000-0002-9536-4522. 


\section{Mediale Integrationsleistungen im Wandel}

Der Artikel untersucht Alltagspraktiken digitaler Teilhabe auf Ebene der Nachbarschaft und der Stadt. Ausgangspunkt der Überlegungen ist die Annahme, dass eine kommunikationswissenschaftliche Erforschung medialer Integrationsleistungen noch stärker interpersonal-öffentliche Kommunikation über digitale Medien in den Blick nehmen sollte, da publizistische Medien und die Kommunikationsaktivitäten gesellschaftlicher Intermediäre (wie Parteien, Verbände und Kirchen) zunehmend an Bedeutung verlieren (Jarren 2018, Neuberger 2018). Gleichzeitig schreibt sich die Digitalisierung immer mehr in den Alltag ein und der Alltag wird zum Medienalltag (Hepp 2008). Fast ohne zeitliche oder räumliche Einschränkung kommunizieren Bürger*innen über digitale Anwendungen mit ihren Mitmenschen, wobei die Grenzen zwischen interpersonal-privater und massenmedial-öffentlicher Kommunikation verschwimmen (Haas \& Brosius 2011, Hepp \& Hasebrink 2013). Wegen dieser Fokusverschiebung der gesellschaftlichen Kommunikation in Richtung der interpersonal-öffentlichen Kommunikation und digitaler Medien sollte künftig noch stärker gefragt werden, ob und in welchen Erscheinungsformen in diesen Bereichen gesellschaftliche Integrationsleistungen erbracht werden. Die Ebene des lokalen Nahraumes ist besonders geeignet, um diesen Fragen nachzugehen, da gesellschaftliche Integration erst auf der Ebene der individuellen Lebenswelt erleb- und erfahrbar wird (Friedland \& McLeod 1999, Gentzel, Nitschke \& Wimmer 2020). In Zeiten der Globalisierung und supranationaler Organisationen scheint eine solche Fokussierung auf das Lokale zunächst kontraintuitiv. Doch da die lokale Ebene eine „wesentliche Sozialisationsinstanz demokratischer Einstellungs- und Verhaltensweisen, eine Quelle zur Stärkung des Gemeinschaftssinns und zur Bekämpfung von Politikverdrossenheit" (Vetter 2011: 2) ist, kann angenommen werden, dass sie darüber auch eine stabilisierende Wirkung auf die Legitimität politischer Strukturen und politischer Prozesse auf höheren Ebenen hat (Dahl 1967).

Ziel des Artikels ist es deshalb, auf Grundlage empirischer Befunde eine Einschätzung des integrativen Potenzials der Alltagspraktiken digitaler Teilhabe auf der lokalen Ebene zu liefern und darüber auch der Forschung zu Integration durch interpersonal-öffentliche Kommunikation zu mehr Aufmerksamkeit seitens der Kommunikationswissenschaft zu verhelfen. Dafür entwickeln wir speziell für die lokale Ebene das zweidimensionale Konzept Alltagspraktiken digitaler Teilhabe, bestehend aus den Dimensionen Vergemeinschaftung und politische Partizipation. Entscheidend für das Konzept ist, dass keine Sondersituationen wie zum Beispiel die Beteiligung an Maßnahmen des Quartiersmanagements oder Wahlen in den Blick genommen werden, sondern das alltägliche Geschehen der Teilhabe betrachtet wird.

Als Startpunkt der empirischen Untersuchung wurden Online-Nachbarschaftsplattformen gewählt. In den letzten Jahren entstand eine wachsende Anzahl solcher Plattformen (Schreiber et al. 2017: 211), deren selbst ausgegebenes Ziel es ist, Nachbarschaften zu revitalisieren und zu einem „lebenswerten Ort“ zu machen (Nebenan.de ohne Jahr). Auch lokale Akteure aus Politik und Verwaltung betonen das demokratische Potenzial der Nachbarschaftsplattformen und geben sie gerne als Beispiel für smarte Bürgerbeteiligung im Rahmen städtischer Digitalisierungsbemühungen an (siehe exemplarisch die Digitalisierungsbemühungen der Städte Dortmund oder Hannover (ohne Jahr)). Die Studie ist deshalb von der Frage geleitet, welche Bedeutung Online-Nachbarschaftsplattformen für Alltagspraktiken digitaler Teilhabe auf Ebene der Nachbarschaft und Stadt haben.

Der Beitrag arbeitet zunächst stadtsoziologische Literatur zu Vergemeinschaftung, die Forschung zur Mediennutzung in lokalen Gemeinschaften (local communities) so- 
wie die kommunikations- und medienwissenschaftliche Partizipationsforschung auf und leitet daraus das Forschungskonzept und Forschungsfragen ab. Anschließend wird die Methodik der standardisierten Inhaltsanalyse und der qualitativen Interviews beschrieben, es werden die empirischen Befunde vorgestellt und es wird ein Fazit gezogen.

\section{Forschungsstand: lokale Vergemeinschaftung und politische Partizipation als mediale Integrationsleistungen}

Obwohl die Erforschung der Integrationsfunktion von Medien in der Kommunikationswissenschaft einen hohen Stellenwert besitzt (Beck: 2013 130) und die Debatte um die integrierende (oder desintegrierende) Wirkung von Medien mit dem Aufkommen von Medieninnovationen (Entstehung des Rundfunks, Kabelkommunikation, Internet) stets aktualisiert und weiterentwickelt wird, steht die Entwicklung eines allgemeinen kommunikationswissenschaftlichen Konzepts zur Analyse von Integration aus (siehe dafür noch immer aktuell Jarren 2000). Um das integrative Potenzial von Online-Nachbarschaftsplattformen empirisch fundiert einschätzen zu können, braucht es deshalb eine Klärung des Integrationsbegriffes. Wir verstehen unter Integration ein komplexes Sozialphänomen, welches sich - mit Rekurs auf die Terminologie Hartmut Essers (2001) auf den Ebenen der Systemintegration (Makroebene) und der Sozialintegration (Mikroebene) vollzieht. Unsere Studie setzt auf Ebene der Sozialintegration an, da wir nach den digitalen Alltagspraktiken fragen, durch welche Bürger*innen in den sozialen und politischen Geltungsraum der lokalen Ebene einbezogen werden. Um den Fokus auf die Praktiken von Bürger*innen begrifflich und konzeptionell abzubilden, verwenden wir den Begriff der „Alltagspraktiken digitaler Teilhabe“. Unter Alltagspraktiken der Teilhabe verstehen wir angelehnt an Wimmer (2017: 248) die alltäglichen Aktivitäten von Bürger\%innen (Hepp \& Pfadenhauer 2014), welche sie in ihrem Alltag in konkreten Situationen ausüben. Sie haben zwar einen spezifischen $Z$ weck, gehen aber nicht zwangsläufig mit einer Teilhabeintention einher (Carpentier 2011). Je nachdem, ob sich die Praktiken auf Teilhabe am sozialen oder politischen Geltungsraum beziehen, werden sie in Praktiken der Vergemeinschaftung und der politischen Partizipation unterteilt. Integration stellt in unserer Terminologie insofern den übergeordneten Begriff dar, der durch das Konzept der Teilhabe mit den Dimensionen „Vergemeinschaftung“ und „politische Partizipation" präzisiert wird.

Die folgenden Unterabschnitte arbeiten die Literatur auf, welche den Zusammenhang zwischen digitalem Mediengebrauch und den Dimensionen Vergemeinschaftung und politische Partizipation herstellt. Der Zusammenhang zwischen digitalen Medien und Partizipation ist sowohl in der politischen Kommunikationsforschung (Kersting 2019, Voss 2014) als auch in der Medienkulturforschung (Carpentier 2011, Hepp \& Pfadenhauer 2014, Wimmer 2015) vergleichsweise gut erforscht und über den Bezug auf die Funktionen gesellschaftlicher Kommunikation für demokratische Gesellschaften an den Integrationsdiskurs angebunden (Weiß \& Jandura 2017). Die kommunikationswissenschaftliche Literatur zum Zusammenhang von Mediennutzung und Vergemeinschaftung hat digitale Medien und den Integrationsaspekt bislang jedoch weniger stark erforscht. Zwar befasst sich auch die deutsche Kommunikationswissenschaft verstärkt mit lokaler Vergemeinschaftung (siehe z. B. die Beiträge in Hepp, Kubitschko \& Marszolek 2018), setzt aber andere Akzente. So wird beispielsweise gefragt, wie Vorstellungen städtischer Gemeinschaft medial inszeniert werden (Marszolek, Robel \& Spanka 2018), oder Städte werden als Gelegenheitsstruktur für die mediale Vergemeinschaftung Jugendlicher konzipiert (Hepp, Simon \& Sowinska 2018). Im angloamerikanischen Raum wird im Forschungsfeld zu local communities (siehe z. B. Friedland \& McLeod 1999, Hoffman \& 
Eveland 2010, McLeod et al. 1996) zwar explizit der Zusammenhang zwischen klassischen Medien und community integration (Vergemeinschaftung) (ebd.) untersucht, aber digitale Medien stehen hier noch nicht im Vordergrund. Deshalb wird im Abschnitt Vergemeinschaftung zusätzlich stadtsoziologische Literatur herangezogen.

\section{1 (Digitale) Praktiken der Vergemeinschaftung}

Das Gegensatzpaar Gemeinschaft und Gesellschaft steht im Zentrum von Schriften, welche konstituierend für soziologisches Denken überhaupt sind. Ferdinand Tönnies (1887 [2021]) gebrauchte es in seiner umfassenden Theorie sozialen Wandels zur Kennzeichnung zweier unterschiedlicher Lebensformen und konzipierte den Übergang von Gemeinschaft zu Gesellschaft als Verlustgeschichte. Max Weber hat im Zuge seiner Dynamisierung der Soziologie durch ihre Rückführung auf den Handlungsbegriff auch das Tönnies'sche Gegensatzpaar umgearbeitet (vgl. Lichtblau 2011: 261ff.). Die Gegenüberstellung von „Vergemeinschaftung “ und „Vergesellschaftung“ beschreibt bei Weber daher ausdrücklich das Handeln von sinnhaft aufeinander bezogenen Individuen. Damit kann der Begriff Vergemeinschaftung genutzt werden, um die Art der durch Handlungen konstituierten sozialen Beziehungen zwischen Individuen näher zu bestimmen. Da der Begriff gleichzeitig immer eine räumliche Dimension hat und damit auf einen konkreten geographischen Ort bezogen ist, eignet er sich gut, um die Art sozialer Beziehungen auf der lokalen Ebene zu kennzeichnen.

Die neuere soziologische Stadtforschung hat die klassischen Konzeptionierungen weiterentwickelt und nutzt häufig eine netzwerktheoretische Variante der Konzepte Sozialintegration, Kohäsion und soziales Kapital, um Gemeinschaft oder Vergemeinschaftung zu erschließen (Jonuschat 2012). Aus kommunikationswissenschaftlicher Perspektive ist diese netzwerktheoretische Variante anschlussfähig, da sie die kommunikativen Verbindungen zwischen den Mitgliedern des Netzwerkes herausstellt. Mit den Arbeiten von Jonuschat (2012) und Schreiber und Kollegen (Becker et al. 2018, Schreiber \& Göppert 2018, Schreiber et al. 2017) liegen Studien vor, die mit der auf Granovetter (1973) zurückgehenden Unterscheidung von „strong ties“ und „weak ties“ die Art der kommunikativen Verbindungen über digitale Medien auf Ebene der Nachbarschaft untersuchen. Sie kommen zu dem Schluss, dass Digitalmedien vor allem lose Beziehungen konstituieren, die dadurch, dass sie in hoher Zahl vorliegen, potenziell Grenzen zwischen sozialen Milieus überbrücken und zur Steigerung von sozialem Kapital führen.

Die netzwerktheoretisch orientierte Forschung ist hilfreich, um die Funktion sozialer Beziehungen für die übergeordnete soziale Ebene zu bestimmen, jedoch bleibt die individuelle Ebene mit der Sinngebung der Individuen eine Leerstelle. Um diese Leerstelle zu füllen, wird auf die kommunikationswissenschaftliche Forschung zu local communities zurückgegriffen. Zwar steht ein Update des Forschungszweiges noch aus und digitale Medien stehen nicht im Vordergrund, aber das theoretische Konzept community integration (Vergemeinschaftung) ist hinsichtlich der individuellen Ebene feiner differenziert. Hoffman und Eveland (2010: 175-177) fassen den Forschungsstand zu community integration zusammen und zeigen, dass affektuelle, kognitive und verhaltensbezogene Aspekte des Begriffes differenziert werden. Obwohl diese Unterscheidung konzeptionell trennscharf erscheint, stellen Hoffman und Eveland (2010: 177) fest, dass variierende Terminologien und uneinheitliche Operationalisierung einen Vergleich der Ergebnisse empirischer Studien erschwert. Ähnlich uneinheitlich sind die Aussagen zum Zusammenhang von community integration und Mediennutzung. McLeod et al. (1996) verstehen Mediennutzung als Antezedens von community integration. Hoffmann und Eveland (2010) hingegen betonen, dass keine verlässliche Aussage über die Richtung des 
Zusammenhangs von Mediennutzung und community integration getroffen werden kann. Interessant hinsichtlich unserer zweiten Teilhabedimension ist, dass politische Partizipation bei McLeod et al. (1996) zwar als „political consequences“ (179) einbezogen wird, aber eben lediglich als Konsequenz von community integration und nicht als eigene Integrationsleistung (siehe nächster Abschnitt).

Vor dem Hintergrund des Forschungsstandes kann unser Verständnis von Vergemeinschaftungspraktiken genauer bestimmt werden. In unserem Konzept zur Erfassung von Alltagspraktiken digitaler Teilhabe schließen wir an das Verständnis von Vergemeinschaftungspraktiken als das sinnhaft aufeinander bezogene Handeln von Individuen an einem geographisch bestimmten Ort an. Um die Art der sozialen Handlungen genauer zu fassen, unterscheiden wir außerdem zwischen Handlungen, die enge und lose Interaktionsbeziehungen konstituieren. Durch die Betonung des Handlungsaspektes stehen die überindividuellen Aspekte der Vergemeinschaftung im Vordergrund, jedoch wird auch berücksichtigt, dass mit den Handlungen auf der individuellen Ebene Kognitionen und Affekte verbunden sind. Eine Bestimmung der Richtung des Zusammenhanges zwischen digitaler Mediennutzung und digitaler Vergemeinschaftung ist für unsere Zwecke nicht notwendig, da sie nicht als voneinander getrennte Kategorien gedacht sind, sondern digitale Vergemeinschaftung als Teilhabe durch Medienhandeln verstanden wird.

\section{2 (Digitale) Praktiken politischer Partizipation}

Prinzipiell lässt sich ein weites von einem engen Verständnis politischer Partizipation unterscheiden. Vor allem in der Politikwissenschaft und klassischen sozialwissenschaftlichen Studien wird häufig ein enges Verständnis genutzt und unter politischer Partizipation staatlich organisierte Bürgerbeteiligung oder rechtlich verfasste und somit verbindliche Partizipationsaktivitäten verstanden (Voss 2014: 9f.). Auch wenn die auf das politische System gerichteten Aktivitäten gesellschaftlicher Intermediäre (NGOs, Kirchen, s. o.) als politische Partizipation gewertet wird, meint Partizipation in diesem klassischen Verständnis Tätigkeiten, die Bürger*innen freiwillig unternehmen, um Entscheidungen des politischen Systems zu beeinflussen (Kaase 1992: 682). Obwohl das enge Verständnis auch in der neueren Forschung zu Online-Partizipation erhalten bleibt (siehe z. B. Kersting 2019), gibt es vermehrt Forderungen, politische Partizipation nicht auf manifeste, politische Handlungen zu beschränken, sondern sie als „Kontinuum verschiedener Formen der Teilhabe" zu betrachten (Wimmer 2017: 248). Ekman und Amnå bezeichnen solche Formen der Partizipation als „pre-political“ oder „standby-political“ (2012: 287). Bezogen auf Diagnosen abnehmender politischer Partizipation formulieren sie:

„(...) [C]itizens actually do a lot of things that may not be directly unequivocally classified as ,political participation', but at the same time could be of great significance for future political activities of a more conventional type. If we are interested in declining levels of political participation, we must not overlook such potentially political forms of engagement.” (ebd.: 287f.)

Darauf aufbauend präsentieren sie eine Typologie, die neben manifesten Formen auch latente Formen politischer Partizipation beinhaltet. Die Dimension der „civil participation” umfasst dabei latente Formen wie „discussing politics and societal issues with friends or on the Internet", , writing to an editor" oder "giving money to a charity" (ebd.: 295). Demgegenüber beschreibt die Dimension „political participation“ manifeste Beteiligungsformen wie „voting in elections“, ,acts of non-voting “", „contacting political 
Tabelle 1: Alltagspraktiken digitaler Teilhabe und Operationalisierung

\begin{tabular}{|c|c|c|c|}
\hline \multicolumn{2}{|r|}{ Dimension } & Operationalisierung & Indikatoren \\
\hline \multirow{4}{*}{ 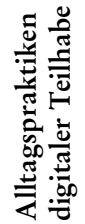 } & \multirow{2}{*}{$\begin{array}{l}\text { Praktiken der Verge- } \\
\text { meinschaftung }\end{array}$} & enge Beziehung & z. B. Sport, Hobbys, Ausflüge \\
\hline & & lose Beziehung & z. B. leihen, tauschen, Tipps \\
\hline & \multirow{2}{*}{$\begin{array}{l}\text { Praktiken der politi- } \\
\text { schen Partizipation }\end{array}$} & manifeste Partizipation & z. B. Wahlaufrufe, Petitionen \\
\hline & & latente Partizipation & $\begin{array}{l}\text { z. B. Diskussionen, } \\
\text { Meinungsäußerungen }\end{array}$ \\
\hline
\end{tabular}

representatives“, „buycotting, boycotting and political consumption“, „signing petitions“ sowie „civil disobedience“ (ebd.).

Obwohl Praktiken politischer Partizipation auch als Spezialform von Vergemeinschaftung oder als Konsequenz von lokaler Integration (siehe voriger Abschnitt) konzipiert werden können, werden sie in unserem Konzept als eigene Dimension behandelt. Für uns unterscheiden sie sich substanziell von Vergemeinschaftungspraktiken, da sie sich direkt oder indirekt auf die im Geltungsraum herbeizuführenden allgemeinverbindlichen Entscheidungen beziehen. Außerdem beziehen sie sich häufig nicht auf ein individuelles Gegenüber, sondern auf den politischen Geltungsraum als abstrakte Größe. Der Umstand, dass mit den Praktiken auf der individuellen Ebene Kognitionen und Affekte verbunden sind, stellt jedoch ebenso wie der Bezug auf einen geographisch bestimmten Ort eine Gemeinsamkeit von Vergemeinschaftung und politischer Partizipation in dem Konzept dar. Um das gesamte Spektrum politischer Partizipation abzudecken, berücksichtigen wir Aktivitäten, die einem engen und weiten Verständnis politischer Partizipation zuzuordnen sind.

\section{Forschungskonzept und Forschungsfragen}

Aufbauend auf diesen Darlegungen operationalisieren wir die Dimensionen Vergemeinschaftung und politische Partizipation im Konzept Alltagspraktiken digitaler Teilhabe über die Unterscheidung „enge Beziehungen“ und „lose Beziehungen“ bzw. „manifeste Partizipation“ und „latente Partizipation“.

- Vergemeinschaftung durch enge Beziehungen geschieht durch intensivere Interaktionsbeziehungen, die meistens ein gemeinsames Interesse beinhalten oder in der gemeinsamen Ausführung von Freizeitaktivitäten bestehen. Diese Beziehungen sind nicht punktuell, sondern überdauern einen gewissen Zeitraum und gehen über das digitale weit hinaus.

- Vergemeinschaftung durch lose Beziehungen geschieht durch flüchtige Interaktionsbeziehungen, in denen beispielsweise ortsspezifisches Wissen und Ressourcen ausgetauscht werden. Viele dieser Interaktionen finden rein online statt oder werden nur durch kurze Begegnungen ergänzt, zum Beispiel, wenn an der Haustür Dinge ausgetauscht werden.

- Als Praktiken manifester Partizipation werden Aktivitäten verstanden, die sich direkt oder indirekt auf die im Geltungsraum herbeizuführenden allgemeinverbindlichen Entscheidungen beziehen.

- Praktiken latenter Partizipation umfassen in Anschluss an Ekman und Amnå (2012) Aktivitäten wie politische Meinungsäußerungen, soziales Engagement und politischen Konsum. Tabelle 1 gibt einen Überblick über die Operationalisierung (Anhang eins bietet eine Übersicht über alle Indikatoren). 
Auf Basis der bisherigen Überlegungen wird die zu Beginn formulierte leitende Fragestellung in zwei Forschungsfragen ausdifferenziert. Forschungsfrage 1 zielt auf eine explorative Vermessung der auf Online-Nachbarschaftsplattformen vorzufindenden Teilhabepraktiken. Forschungsfrage 2 bezieht zusätzlich die Wahrnehmung der Nutzer*innen der Plattformen mit ein, wodurch affektive und kognitive Aspekte auf der individuellen Ebene erfasst werden.

Forschungsfrage 1: In welchem Umfang und in welcher Form werden Online-Nachbarschaftsplattformen für Alltagspraktiken digitaler Teilhabe genutzt?

Forschungsfrage 2: Wie nebmen Nutzer*innen die Online-Nachbarschaftsplattformen binsichtlich der Alltagspraktiken digitaler Teilhabe wabr?

Um Forschungsfrage $1 \mathrm{zu}$ beantworten, wurde eine standardisierte Inhaltsanalyse der Postings einer Online-Nachbarschaftsplattform vorgenommen. Zur Beantwortung von Forschungsfrage 2 wurden qualitative Interviews mit Nutzer\%innen der Plattform geführt.

\section{Methode: Standardisierte Inhaltsanalyse und qualitative Interviews}

\subsection{Sampling}

Nach einer Recherche bundesweiter und lokaler Nachbarschaftsplattformen wurde die Plattform nebenan.de ausgewählt. Mit ca. 1,5 Millionen aktiven Nutzer־innen ist sie die größte Nachbarschaftsplattform Deutschlands und setzt sich aufgrund von Marktkonzentration und Netzwerkeffekten immer stärker gegen Wettbewerber durch. Für die Inhaltsanalyse wurde exemplarisch die Stadt Augsburg als Untersuchungsgebiet gewählt, die auf nebenan.de in insgesamt $32 \mathrm{Nachbarschaften} \mathrm{unterteilt} \mathrm{ist.} \mathrm{Dies} \mathrm{hat} \mathrm{den}$ Vorteil, dass in dem relativ überschaubaren Raum einer mittelgroßen Stadt (ca. 300.000 Einwohner*innen) agiert wird und die Forscher*innen über regionale Vorkenntnisse verfügten. Die Auswahl der Befragten für die qualitativen Interviews erfolgte in einem Schneeballverfahren. Ausgangspunkt waren Nutzer*innen der Plattform, die während der standardisierten Inhaltsanalyse dadurch auffielen, dass sie sehr aktiv posteten oder selbst eher passiv waren, aber auf Postings anderer reagierten. In allen Interviews wurden die Befragten gebeten, weitere Personen zu nennen, die für ein Interview zur Verfügung stehen könnten. Bei der Auswahl der Befragten wurde versucht, Personen zu akquirieren, die möglichst aus unterschiedlichen Nachbarschaften stammen. Die Auswahl erfolgte bis zur theoretischen Sättigung.

\subsection{Datenerhebung und Analyse}

Das Kodierbuch für die standardisierte Inhaltsanalyse wurde auf Basis des theoretischen Konzepts entlang der Dimensionen Vergemeinschaftung und politische Partizipation erstellt. Die Indikatoren für die Dimension politische Partizipation wurden in Anlehnung an die beiden Dimensionen civil participation (latent) und political participation (manifest) aus der Typologie von Ekman und Amnå (2012) entwickelt (vgl. für eine erste empirische Operationalisierung auch Wimmer 2017: 254). Da diese Typologie das Ergebnis einer Zusammenfassung der in der Partizipationsforschung verwendeten Indikatoren ist, schien sie gut geeignet, um das gesamte Spektrum von Partizipationspraktiken abzubilden. Diese Einschätzung hat sich in der Testphase des Kodierbuchs bestätigt, da keine weiteren Indikatoren zur politischen Partizipation aufgenommen werden mussten. 
Für die Dimension Vergemeinschaftung lag kein vergleichbar gut ausgearbeiteter Katalog an Indikatoren vor. Die Operationalisierungen in Studien zu community integration sind uneinheitlich (siehe oben) und beziehen sich außerdem häufig auf kognitive oder affektuelle Aspekte von Vergemeinschaftung (vgl. Hoffman \& Eveland 2010: 175177). Die Studie von Schreiber et al. (2017: 213) nennt einige Aktivitäten (Kleinanzeigen, Hilfsleistungen, Hinweise, Freundschaften und Veranstaltungen), an denen lose und enge Beziehungen festgemacht werden können, allerdings ohne eine systematische $\mathrm{Ty}$ pologie zu liefern. Die bei Schreiber et al. (2017) genannten Aktivitäten wurden als Ausgangspunkt für die Dimension Vergemeinschaftung genutzt. In der Testphase des Kodierbuchs wurden diese Indikatoren jedoch verfeinert und es wurden zusätzliche Indikatoren aus dem Testmaterial heraus entwickelt. Außerdem zeigte sich in der Testphase, dass Aktivitäten mit rein gewerblichem Charakter einen großen Teil der Gesamtinteraktion auf der Plattform ausmachen, weshalb eine Zusatzkategorie Gewerbliches (mit Unterkategorien) zur Erfassung dieser sonstigen gewerblichen Aktivitäten geschaffen wurde. Zuerst wurde überlegt, diese Aktivitäten in die anderen Dimensionen zu integrieren, da auch die Konstrukte Vergemeinschaftung und politische Partizipation Indikatoren mit einer ökonomischen Dimension beinhalten (Austausch von Ressourcen, politischer Konsum). Die in der Testphase bemerkten gewerblichen Aktivitäten unterscheiden sich aber konzeptionell von den anderen Dimensionen, da sie allein der Generierung von Einkommen oder dem Abschluss von Verträgen (Immobilien) dienen und sich nicht auf den sozialen oder politischen Geltungsraum beziehen.

Das Kodierbuch differenziert insgesamt 45 Aktivitäten, die sich über die beiden Dimensionen und die Zusatzkategorie verteilen. Nach intensiver Schulung der Kodierer*innen konnte im dritten Durchgang eine zufriedenstellende Intercoder-Reliabilität erreicht werden (Krippendorff's Alpha $=.84$, drei Kodierer*innen). Für den Reliabilitätstest wurden alle Postings im Verlauf einer Woche kodiert (Durchgang eins: 76 Postings, Durchgang zwei $=122$ Postings, Durchgang drei $=99$ Postings). Die Datenerhebung erstreckte sich über zwölf Wochen (20.01.2020-12.04.2020). Innerhalb dieses Zeitraumes wurden alle nutzergenerierten Postings (insgesamt 1.197) aus allen 32 Nachbarschaften der Stadt berücksichtigt und einer Aktivität zugeordnet. Der Zugriff auf die Postings erfolgte über persönliche nebenan.de-Profile der an der Studie beteiligten (und in Augsburg wohnhaften) studentischen Hilfskräfte und wissenschaftlichen Mitarbeiter*innen.

Während der Erhebung spitzte sich die Covid-19-Pandemie zu und im Stadtgebiet galt eine Ausgangssperre. Um abzuschätzen, ob sich durch die Pandemie die Aktivitäten auf der Plattform verändern, wurde deshalb zusätzlich zur Kategorisierung in die zwei Dimensionen und die Zusatzkategorie erhoben, ob sich das Posting auf die Pandemie bezieht. Im Abschnitt Limitationen wird abgeschätzt, inwiefern die Covid-19-Situation einen Einfluss auf die Ergebnisse hat.

Die Erhebung der Wahrnehmung der Nutzer*innen erfolgte nach Abschluss der Auswertung der standardisierten Inhaltsanalyse durch 20 qualitative Leitfadeninterviews (12.08.2020-29.09.2020). Die Interviewerin nutzte in flexibler Handhabung einen Interviewleitfaden. Der Leitfaden enthielt folgende Fragenblöcke: Bezug der Person zur Stadt, Vergemeinschaftung, politische Partizipation, Relevanz der lokalen Begrenzung der Plattform, Veränderungen während des Lockdowns und weitere Medien zum Erleben der Stadt. Es wurde in den Interviews nicht zwischen manifester und latenter Partizipation und engen und losen Kontakten unterschieden, da dies externe Analysekriterien der Forscher*innen sind, die nicht auf der subjektiven Wahrnehmung der Nut- 
zer*innen basieren. Alle Interviews wurden aufgezeichnet und mittels eines einfachen Transkriptionssystems (Dresing \& Pehl 2015) verschriftlicht.

In einer deskriptiven Analyse wurde zunächst dargestellt, auf welche Dimensionen sich die geposteten Beiträge verteilen, und Längsschnitts-Analysen lieferten Rückschlüsse auf den Einfluss bestimmter Ereignisse auf das tägliche Posting-Verhalten. Die Analyse der qualitativen Interviews erfolgte durch eine qualitative Inhaltsanalyse, wobei die ersten beiden Schritte des Vorgehens nach Kelle und Kluge vollzogen wurden (2010). Die Frageblöcke des Interviewleitfadens bildeten die Hauptkategorien und die einzelnen Fragen dienten als Unterkategorien. Wenn notwendig, wurde eine weitere Ebene hinzugefügt.

\subsection{Methodische Limitationen}

Die Datenerhebung für die Inhaltsanalyse erfolgte zwischen dem 20. Januar und 12. April 2020, also dem Zeitraum, in dem sich die Covid-19-Pandemie entwickelte und schließlich der erste Lockdown verhängt wurde. Um abzuschätzen, ob sich durch die Pandemie und den Lockdown die Aktivitäten auf der Plattform veränderten, wurde deshalb erhoben, ob sich das jeweilige Posting auf die Pandemie bezieht (siehe oben). Es zeigte sich, dass die Aktivitäten insgesamt kurzzeitig stark anstiegen, aber auch schnell wieder abfielen. Der Vergleich im Zeitverlauf zeigt außerdem, dass sich das Verhältnis der Teilhabedimensionen zueinander vor, während und nach dem kurzzeitigen Anstieg kaum veränderte. Insofern erscheinen die Daten durchaus generalisierbar und sind durch die Covid-19-Pandemie nicht systematisch verzerrt. Das gleiche nehmen wir für die qualitativen Interviews an, da die Entwicklungen der Pandemie und insbesondere die Situation während des Lockdowns gesondert behandelt wurden.

\section{Empirische Befunde}

\subsection{Befunde der quantitativen Vermessung: Vergemeinschaftung über lose Kontakte}

Die standardisierte Inhaltsanalyse zeigt, dass die Plattform primär für Vergemeinschaftung durch lose Kontakte und für gewerbliche Tätigkeiten genutzt wird. Politische Partizipation ist wenig zu finden. 61,5 Prozent aller Postings entfallen auf Vergemeinschaftung, gefolgt von 28,7 Prozent im Bereich Gewerbliches und 7,7 Prozent im Bereich politische Partizipation (Tabelle 2). Innerhalb der Dimension Vergemeinschaftung entfallen 21,5 Prozent der Postings in die Kategorie enge und 78,5 Prozent in die Kategorie lose Kontakte. Bei politischer Partizipation überwiegt latente Partizipation (76 \%) im Vergleich zu manifester Partizipation (24\%). Wird die Verteilung der Praktiken innerhalb der Dimension politische Partizipation betrachtet, zeigt sich, dass Meinungsäußerungen mit Abstand die häufigste Praktik sind (38,04 \%), gefolgt von Aufrufen, gemeinnützig tätig zu werden (27,17\%), und Aufrufen zu Demonstrationen oder anderen politischen Veranstaltungen (16,3 \%). Sieben Praktiken im Bereich politische Partizipation wurden kein einziges Mal kodiert (Aufruf zum Wahlboykott, Wahlkampf, Beschwerde äußern, Aufruf zur Wahlbeteiligung, Aufruf zu zivilem Ungehorsam, Produkte kaufen, Produkte boykottieren). 
Tabelle 2: Aktivitäten auf der Nachbarschaftsplattform insgesamt und innerhalb der Dimensionen

\begin{tabular}{|c|c|c|}
\hline \multicolumn{3}{|c|}{ Aktivitäten auf der Nachbarschaftsplattform $(\mathrm{N}=1.197)$} \\
\hline \multirow[b]{2}{*}{ Vergemeinschaftung } & \multicolumn{2}{|c|}{$61,48 \%(n=736)$} \\
\hline & $\begin{array}{c}\text { davon lose Kontakte: } 78 \% \\
(\mathrm{n}=574)\end{array}$ & $\begin{array}{c}\text { davon enge Kontakte: } 22 \% \\
(\mathrm{n}=162)\end{array}$ \\
\hline Gewerbliches & \multicolumn{2}{|c|}{$28,65 \%(n=343)$} \\
\hline \multirow[b]{2}{*}{ Politische Partizipation } & \multicolumn{2}{|c|}{$7,69 \%(n=92)$} \\
\hline & $\begin{array}{c}\text { davon latent: } 76 \% \\
(\mathrm{n}=70)\end{array}$ & $\begin{array}{c}\text { davon manifest: } 24 \% \\
(\mathrm{n}=22)\end{array}$ \\
\hline Sonstiges & \multicolumn{2}{|c|}{$2,17 \%(n=26)$} \\
\hline
\end{tabular}

Werden die zehn häufigsten Praktiken betrachtet (Tabelle 3), zeichnet sich das Bild einer Plattform, auf der Nachbar*innen sich Gegenstände schenken und leihen, Gebrauchtes zum Kauf anbieten oder Dinge kaufen möchten, Wohnungen zur Miete gesucht und Tipps und Hilfe ausgetauscht werden.

\section{Tabelle 3: Top-Ten-Aktivitäten auf der Nachbarschaftsplattform}

\begin{tabular}{lc}
\hline Aktivitäten & Relative Häufigkeit $(\mathbf{N}=1.197)$ \\
\hline 1. Schenken (Vergemeinschaftung) & $10,94 \%(\mathrm{n}=131)$ \\
2. Hilfe angeboten (Vergemeinschaftung) & $8,19 \%(\mathrm{n}=98)$ \\
3. Kaufnachfrage (Gewerbliches) & $8,19 \%(\mathrm{n}=98)$ \\
4. Hilfe gesucht (Vergemeinschaftung) & $7,10 \%(\mathrm{n}=85)$ \\
5. Tipps gesucht (Vergemeinschaftung) & $6,68 \%(\mathrm{n}=80)$ \\
6. Immobilie mieten (Gewerbliches) & $5,51 \%(\mathrm{n}=66)$ \\
7. Tipps gegeben (Vergemeinschaftung) & $5,18 \%(\mathrm{n}=62)$ \\
8. Flohmärkte (Gewerbliches) & $4,76 \%(\mathrm{n}=57)$ \\
9. Kaufangebot (Gewerbliches) & $4,68 \%(\mathrm{n}=56)$ \\
10. Leihen (Vergemeinschaftung) & $4,43 \%(\mathrm{n}=53)$ \\
\hline
\end{tabular}

Als Antwort auf Forschungsfrage 1 und als Hauptbefund der standardisierten Inhaltsanalyse lässt sich also festhalten, dass sich Teilhabe auf Online-Nachbarschaftsplattformen vor allem in Form von Vergemeinschaftungspraktiken zeigt und nicht in Form politischer Partizipation. Zudem überwiegen in den Postings deutlich Vergemeinschaftungspraktiken, durch welche sich lose Beziehungen konstituieren und keine engen Beziehungen.

Im nächsten Abschnitt werden die Befunde der standardisierten Analyse durch die qualitativen Interviews ergänzt, wodurch sich wichtige Ergänzungen und Präzisierungen ergeben. 


\subsection{Befunde der qualitativen Befragung: Vergemeinschaftung durch Beobachtung und die Plattform als "Introduction Service“"}

Auch in den qualitativen Interviews erscheint die Plattform als Ort, an dem vor allem lose Kontakte geknüpft werden. Die Nutzer־innen berichten, dass in ihrer Wahrnehmung der Austausch von ortsspezifischem Wissen und Ressourcen einen wichtigen Teil der Kommunikation ausmacht. Die Interaktionen, die zu den losen Kontakten zählen, finden hauptsächlich rein online statt, das heißt in den Postings, Kommentaren und Gruppenbereichen der Plattform, oder beinhalten nur kurze Begegnungen, zum Beispiel beim Austausch von Dingen an der Haustür. Dieser Befund lässt sich gut in Übereinstimmung mit den Top-Ten-Aktivitäten auf der Plattform bringen, wo der Austausch von Dingen und ortsspezifischen Informationen dominiert. Allerdings dürfen diese Transaktionen nicht auf ihren rein zweckmäßigen Charakter reduziert werden, denn der Austausch hat auch ein affektives Moment, durch welches Gefühle der Hilfsbereitschaft und der Zusammengehörigkeit erzeugt werden. Wir deuten dies so, dass schon die kurzen Sozialkontakte die für Städte typische Anonymität in Nachbarschaften mildern. In einem Interview heißt es dazu: „Und manchmal redet man eben kurz. Und wenn man auf der Straße dann das Gesicht sieht und grüßt, ist das schon nett familiär so in der Nachbarschaft" (Interview 12, Miran; alle Namen sind geändert).

Ein für uns zentraler Aspekt für das Verständnis der Vergemeinschaftungsprozesse durch die Plattform ist, dass die gefühlte Zugehörigkeit und Verbindung zur Nachbarschaft auch durch passives Beobachten geschehen kann. Es scheint nicht unbedingt notwendig, dass Nachbar*innen die Gemeinschaft anderer aktiv durch eigene Beiträge auf der Plattform suchen, sondern schon das Beobachten der Kommunikation von Nachbar*innen kann ein Zugehörigkeitsgefühl im Sinne einer „public familiarity“ (Blokland $\&$ Nast 2014) erzeugen (vgl. dazu auch Becker et al. 2018: 208). Es ist anzunehmen, dass diese Vergemeinschaftung durch Beobachtung auf Nachbarschaftsplattformen besonders gut funktioniert, da die Kommunikation durch Klarnamen und die Angabe des Wohnortes weniger anonym ist als beispielsweise in lokalen Facebook-Gruppen.

In der standardisierten Inhaltsanalyse erscheint es so, als ob die Etablierung und Aufrechterhaltung enger Kontakte kaum eine Rolle auf der Plattform spielt. Die qualitativen Interviews zeigen jedoch, dass die Plattform stärker als „Introduction Service“ (Wellman \& Hampton 1999: 562) dient, als es in der standardisierten Inhaltsanalyse den Anschein hat, da sie vor allem zur Anbahnung von engen Kontakten genutzt wird. In der Inhaltsanalyse sind diese engen Kontakte schlecht zu erfassen, da über die Plattform häufig nur Sozialkontakte angebahnt werden und der Großteil der Kommunikation anschließend Face-to-Face oder vermittelt über andere Medien der interpersonalen Kommunikation (vor allem Messenger Dienste) stattfindet. Die Plattform schafft über die Sichtbarmachung von Interessen, Zielen und gemeinsamen Anliegen eine Konnektivität im Sinne von van Dijck und Poell (2013: 13) und verbindet Nachbar*innen, die zwar viel gemeinsam haben, sich aber vorher unbekannt waren. Dieser Befund ist insofern interessant, da die Entstehung von interessensbasierten Netzwerken in der frühen Netzwerkforschung als „networked individualism“ (Wellman 2001) verstanden wurde, der gleichzeitig die Loslösung aus tradierten Netzwerken wie der Nachbarschaft oder der Familie bedeutet. Eine solche Entkopplung von interessenbasiertem Netzwerk und tradiertem ortsbezogenem Netzwerk findet auf der lokalen Ebene nicht statt, da sich beide Netzwerkmechanismen verschränken. Ob die so entstehenden Netzwerke stabiler sind oder andere Qualitäten aufweisen als Netzwerke, die nur auf einem Mechanismus beruhen, kann auf Grundlage der erhobenen Daten nicht bewertet werden. 
Ebenfalls nicht abschließend abgeschätzt werden kann die Frage, ob es durch die engen Kontakte in den untersuchten Nachbarschaften zur Überbrückung sozialer Milieus und damit zu Bridging Social Capital im Sinne Putnams (2000) kommt. Es scheint jedoch so, dass die Plattform durchaus Personen unterschiedlicher Altersgruppen und Herkunft vernetzt. Cemal, ein Befragter türkischer Herkunft, der neu in die Stadt gezogen ist, berichtet von einer Bekanntschaft:

„So, there is one person, also from the group I met. He is living alone, he is lonely. And he is a little bit aged people. Around 70 . He has problem with the legs. So, he needs person or people to talk to him every day. So, I met this man also one time / for the first time. Later I have introduced two more people to this man to a friend or Bekannten to communicate with other people, with other of my friends. So, they also improving German. Meanwhile he also spends times with this people. So this is also a kind of also solution / Lösung for the / how do you call it / loneliness". (Interview 15, Cemal).

Auch Henriette (63 Jahre, vor zehn Jahren in die Stadt gezogen) berichtet von einem regelmäßigen Stammtisch, den sie über die Plattform organisiert:

„Und so ist praktisch meine Sache entstanden und ich bin auch ganz froh, dass ich die Gruppe habe, und ich hab auch ganz, ganz nette Leute bei / also das Alter war, die erste, die da war, mit, die war damals 18 Jahre bis zu drei / 88 war die Älteste“ (Interview 13, Henriette).

In der Dimension politische Partizipation zeichnen die Ergebnisse der Interviews wieder ein ähnliches Bild wie bei der Inhaltsanalyse. Etwa die Hälfte der Befragten gibt an, dass sie auf der Plattform noch nie oder nur vereinzelt Beiträge wahrgenommen hat, die in die Dimension manifeste oder latente politische Partizipation fallen. Wenn politische Inhalte wahrgenommen werden, handelt es sich fast ausschließlich um Meinungsbeiträge, in denen das aktuelle politische Zeitgeschehen kommentiert wird. Auffällig ist, dass die Befragten, denen politische Inhalte auffallen, diese häufig negativ bewerten und die Plattform nicht als geeigneten Ort für politische Diskussionen beschreiben. Kritisiert wird vor allem, dass die Mehrzahl der politischen Beiträge einen rechtspopulistischen Hintergrund hat und sich nicht auf Lokalpolitik bezieht, wodurch politische Diskussionen schnell in Richtung polarisierender Themen ohne lokalen Bezug abrutschen. Einige Nachbar*innen sehen die Gefahr, dass politische Diskussionen auf der Plattform das Gemeinschaftliche in der Nachbarschaft gefährden. Eleonore (53 Jahre, seit sechs Jahren in der Stadt, vorher wohnhaft im Nachbarort) berichtet, sie wolle auf der Plattform und in der Nachbarschaft lieber keine politischen Diskussionen führen, da „das nachbarschaftliche Verhältnis gestört werden könnte, wenn die politischen Einstellungen zu weit auseinanderliegen "(Interview 7, Eleonore). Gleichzeitig geben die Befragten an, dass sie gerne online Diskussionen zu lokalen Themen führen würden, die in geeigneter Weise moderiert werden. Die Interviews geben schließlich Hinweise darauf, dass für lokale politische Partizipation andere Plattformen relevant sind. Immer wieder wird auf lokale Facebook-Gruppen verwiesen, die von lokalen politischen Initiativen betrieben werden und die dem Austausch und der Mobilisierung dienen. Außerdem haben Messenger wie Telegram und WhatsApp eine steigende Bedeutung, sowohl für die externe Kommunikation und Mobilisierung als auch für die interne Kommunikation und Organisation.

\section{Fazit}

Der Beitrag war von der Frage geleitet, welche Bedeutung Online-Nachbarschaftsplattformen für Alltagspraktiken digitaler Teilhabe haben, und es sollte eine Einschätzung des integrativen Potenzials der Plattformen erfolgen. Für uns zeichnen die Befunde ins- 
gesamt ein positives Bild, allerdings muss zwischen den Dimensionen differenziert werden. Integration vollzieht sich auf den und durch die Plattformen vor allem in Form von Vergemeinschaftung. Auf der Plattform werden lose Beziehungen gepflegt, durch die ortsspezifisches Wissen und Ressourcen ausgetauscht werden. Es werden enge Beziehungen zu Menschen mit gemeinsamen Interessen und Bedürfnissen vermittelt, und das Zugehörigkeitsgefühl zur Nachbarschaft kann auch durch das alleinige Beobachten von Vergemeinschaftungspraktiken anderer entstehen.

Den Umstand, dass Plattformen Vergemeinschaftung bereits durch Beobachtung der Vergemeinschaftung anderer ermöglichen, sehen wir als möglichen Anknüpfungspunkt an die Öffentlichkeitsforschung. Die politisch orientierte Öffentlichkeitsforschung nimmt an, dass es die durch publizistische Medien konstituierte Öffentlichkeit ist, die es Bürger*innen erlaubt, sich als „Rechtsgemeinschaft" (Imhof 2013: 10) wahrzunehmen, was eine Bedingung für die Ausübung von Bürgerrechten ist (Eisenegger \& Udris 2019: 6). Anders ausgedrückt, ist die Selbstwahrnehmung als Mitglied eines Geltungsbereiches, der prinzipiell allen offensteht, die Voraussetzung der Konstitution einer Bürgerschaft für sich (Weiß \& Jandura 2017). Weitere Theoriebildung zum Konnex von Medien, Öffentlichkeit und Integration sollte diskutieren, inwiefern medial hergestellte Vergemeinschaftung für demokratische Gesellschaften eine ähnliche Funktion haben kann wie die durch publizistische Medien hergestellte Öffentlichkeit. Denn ebenso wie Öffentlichkeit es tut, erlauben auch lokale Vergemeinschaftungsprozesse es Bürger*innen, sich als Teil eines lokalen Geltungsraumes wahrzunehmen. Es ist außerdem anzunehmen, dass die Selbstwahrnehmung als Teil einer Gemeinschaft in positiver Wechselwirkung zu anderen Prozessen der gesellschaftlichen Integration in dem jeweiligen lokalen Geltungsraum steht. Einschränkend ist sicherlich zu berücksichtigen, dass diese Funktion von interpersonal-öffentlicher Kommunikation für Geltungsräume oberhalb der lokalen Ebene unwahrscheinlicher ist. Denn auf der lokalen Ebene drängt sich für Nutzer*innen von Social-Media-Plattformen ein Vergleich der beobachteten Praktiken anderer mit der eigenen Lebenswelt stärker auf, da durch den geteilten Lebensraum eine Gemeinsamkeit hergestellt wird. Außerdem bleibt nur auf der lokalen Ebene die Entkopplung von interessensbasierten Netzwerken und ortsbasierten Netzwerken aus.

Das positive Fazit zum integrativen Potenzial der Plattformen muss in Bezug auf politische Partizipation jedoch abgeschwächt werden. Der Umfang an Partizipationspraktiken ist gering und wird zudem ambivalent bewertet. Zwar wünschen sich einige Nutzer*innen einen Online-Ort, an dem über Lokalpolitik diskutiert wird, sehen aber die Nachbarschafts-Plattform als ungeeigneten Ort dafür an. Daraus sollte aber nicht der Schluss gezogen werden, dass digitale Medien der interpersonal-öffentlichen Kommunikation generell keine Relevanz für lokale Partizipation haben. Künftige Forschung zur lokalen politischen Partizipation sollte stattdessen andere Medien in den Blick nehmen: Facebook, Telegram und WhatsApp sind auch im lokalen Kontext die Orte, an denen politische Meinungsäußerung stattfindet, Bürgergruppen sich organisieren und lokale Akteure strategisch kommunizieren. Es ist jedoch nicht verwunderlich, dass es bisher nur wenige Studien in diesem Bereich gibt, denn die Hindernisse (methodische und ethische Standards) bei der Erforschung dieser Grenzbereiche der öffentlichen Kommunikation sind hoch. Es lohnt sich jedoch, und die Entwicklung von Forschungsdesigns für diesen Bereich ist unumgänglich.

Ein auffälliger Befund der standardisierten Inhaltsanalyse war außerdem, dass Tätigkeiten mit gewerblichem Charakter einen hohen Anteil auf der Plattform haben. Zusammengenommen mit dem Umstand, dass auch einzelne Aktivitäten in den Teilhabedimensionen Vergemeinschaftung und politische Partizipation eine ökonomische Di- 
mension beinhalten (Austausch von Ressourcen, politischer Konsum), muss diskutiert werden, ob die gewerblichen Aktivitäten in unser theoretisches Konzept integriert werden müssen. Im Rahmen dieser Studie haben wir uns dagegen entschieden, da hier die Sozialintegration in den sozialen (Vergemeinschaftung) und politischen (Partizipation) Geltungsraum auf der lokalen Ebene interessiert. Vergemeinschaftungspraktiken, wie wir sie untersucht haben, bestehen aus sozialen Beziehungen, in denen sich Bürger٪innen gerade jenseits der Rollen begegnen, die mit bestimmten gesellschaftlichen Teilbereichen verbunden sind (zum Beispiel Käufer/Verkäufer). Da Aktivitäten in der Kategorie Gewerbliches allein der Generierung von Einkommen oder dem Abschluss von Verträgen dienen, basieren diese Interaktionen jedoch gerade auf den hochgradig wechselseitig typifizierten Verhaltensmustern, wie sie für die Ökonomie typisch sind. Auch eine Zurechnung zur politischen Partizipation erscheint wenig hilfreich, da kein Bezug zur Herbeiführung allgemeinverbindlicher Entscheidungen oder zum Ausüben grundlegender Kommunikationsfreiheiten (Meinungsäußerung, Verbreitungsfreiheit) besteht.

Schließlich sehen wir auch eine normative Dimension unserer Befunde. Wir verstehen sie als Beitrag zur Debatte um die Frage nach einem Gestaltungsauftrag der Politik hinsichtlich der lokalen Medienlandschaft (vgl. Jarren 2018). Die interpersonal-öffentliche Kommunikation über digitale Medien breitet sich aus, und es zeigt sich, dass auch diese Medien auf lokaler Ebene wichtige demokratische Funktionen erfüllen können. Deshalb darf die Ausgestaltung der Kommunikationsumgebungen in diesem Bereich nicht den Anbietern der digitalen Angebote überlassen werden. Dies erscheint umso dringlicher angesichts der Ausdünnung und der schwindenden Vielfalt des Zeitungsangebots auf der lokalen Ebene (Röper 2020). Insofern braucht es neue Leitbilder für die Ausgestaltung der lokalen Medienlandschaft, denn die demokratische Gesellschaft benötigt lebendige Kommunikationsgemeinschaften auf allen Ebenen.

\section{Literatur}

Beck, K. (2013): Integration. In G. Bentele, H.-B. Brosius, \& O. Jarren (Hrsg.), Lexikon der Kommunikations- und Medienwissenschaft (S. 129-130), Wiesbaden: Springer VS.

Becker, A., Göppert, H., Schnur, O., \& Schreiber, F. (2018): Die digitale Renaissance der Nachbarschaft. FWS, 2018 (4), S. 206-210.

Blokland, T., \& Nast, J. (2014): From Public Familiarity to Comfort Zone: The Relevance of Absent Ties for Belonging in Berlin's Mixed Neighbourhoods. International Journal of Urban and Regional Research, 38(4), S. 1142-1159.

Carpentier, N. (2011): Media and Participation. A Site of Ideological-Democratic Struggle. Bristol: Intellect.

Dahl, R. A. (1967): The Problem of Civic Competence. American Political Science Review, 1967(61), S. 953-970.

Dresing, T., \& Pehl, T. (2015): Praxisbuch Interview. Transkription \& Analyse. Marburg: Dr. Dresing und Pehl GmbH.

Eisenegger, M., \& Udris L. (2019): Eine öffentlichkeitssoziologische Theorie des sozialen Wandels in der digitalen Gesellschaft. In M. Eisenegger, L. Udris, \& P. Ettinger (Hrsg.), Wandel der Öffentlichkeit und der Gesellschaft: Gedenkschrift für Kurt Imbof (S.3-28). Wiesbaden: Springer VS.

Ekman, J., \& Amnå, E. (2012): Political Participation and Civic Engagement: Towards a New Typology. Human Affairs, 22(3), 283-300, https://doi.org/10.2478/s13374-1.

Esser, H. (2001): Integration und ethnische Schichtung (= Arbeitspapier Nr. 40). Mannheim: Mannheimer Zentrum für Europäische Sozialforschung (MZES).

Friedland, L., \& McLeod, J. (1999): Community Integration and Mass Media: A Reconsideration. In: D. Demers, \& K. Viswanath (Hrsg.), Mass Media, Social Control and Social Change. A Macroperspective (S.197-226). Iowa: Iowa State University Press. 
Gentzel, P., Nitschke, P., \& Wimmer, J. (2020): Integration durch digitale Urbanität? Die Digitale Stadt als Forschungsfeld der Kommunikationswissenschaft. In V. Gehrau, A. Waldherr, \& A. Scholl (Hrsg.), Integration durch Kommunikation: Jabrbuch der Publizistik- und Kommunikationswissenschaft 2019 (S. 167-177). Münster: DGPuK.

Granovetter, M. (1973): The Strength of Weak Ties. American Journal of Sociology (78), S. 13601380.

Haas, A., \& Brosius, H.-B. (2011): Interpersonal-öffentliche Kommunikation in Diskussionsforen. Strukturelle Äquivalenz mit der Alltagskommunikation? In J. Wolling, A. Will, \& C. Schumann (Hrsg.), Medieninnovationen. (S. 103-119). Konstanz: UVK.

Hepp A. (2008): Netzwerke der Medien - Netzwerke des Alltags: Medienalltag in der Netzwerkgesellschaft. In T. Thomas (Hrsg.), Medienkultur und soziales Handeln (S. 63-89). Wiesbaden: VS Verlag für Sozialwissenschaften.

Hepp, A., \& Hasebrink, U. (2013): Human Interaction and Communicative Figurations. The Transformation of Mediatized Cultures and Societies. Communicative Figurations, Working Paper No. 2. https://www.uni-bremen.de/fileadmin/user_upload/fachbereiche/fb9/zemki/ media/photos/publikationen/working-papers/2013/CoFi_EWP_No-2_Hepp_Hasebrink.pdf [27.07.2021].

Hepp, A., Kubitschko, S., \& Marszolek. I. (Hrsg.) (2018): Die mediatisierte Stadt: Kommunikative Figurationen des urbanen Zusammenlebens. Wiesbaden: Springer VS.

Hepp, A., \& Pfadenhauer, M. (2014): Mediatisierte Partizipation? In F. Krotz, C. Despotović, \& M.-M. Kruse (Hrsg.), Die Mediatisierung sozialer Welten. Synergien empirischer Forschung (S. 235-262). Wiesbaden: Springer VS.

Hepp, A., Simon, P., \& Sowinska, M. (2018): Zusammenleben in der mediatisierten Stadt. Die kommunikativen Figurationen der urbanen Vergemeinschaftung junger Menschen. In A. Hepp, S. Kubitschko, \& I. Marszolek (Hrsg.), Die mediatisierte Stadt: Kommunikative Figurationen des urbanen Zusammenlebens (S. 89-119). Wiesbaden: Springer VS.

Hoffman, L. H., \& Eveland, W. P. (2010): Assessing Causality in the Relationship Between Community Attachment and Local News Media Use. Mass Communication and Society, 13(2), 174-195, https://doi.org/10.1080/15205430903012144.

Imhof, K. (2013): Die Demokratie, die Medien und das Jahrbuch. In fög/Universität Zürich (Hrsg.), Jabrbuch 2012. Qualität der Medien - Schweiz - Suisse - Svizzera (S. 9-11). Basel: Schwabe.

Jarren, O. (2000): Gesellschaftliche Integration durch Medien? Medien E Kommunikationswissenschaft $M \mathcal{E} K, 48(1), 22-41$, https://doi.org/10.5771/1615-634x-2000-1-22.

Jarren O. (2018): Öffentlichkeit(en) im Wandel: Rede auf dem vhw Verbandstag 2018. FWS, 2018(6), 253-254.

Jonuschat, H. (2012): The Strength of Very Weak Ties - Lokale soziale Netze in Nachbarschaften und im Internet. [Dissertation, vorgelegt an der HU Berlin]. http://edoc.hu-berlin.de/disser tationen/jonuschat-helga-2012-06-12/PDF/jonuschat.pdf [20.07.2021].

Kaase, M. (1992): Partizipation. In D. Nohlen (Hrsg.), Wörterbuch zur Politik (4. Aufl., S. 682-684). München: Piper.

Kelle, U., \& Kluge, S. (2010): Vom Einzelfall zum Typus: Fallvergleich und Fallkontrastierung in der qualitativen Sozialforschung. Wiesbaden: VS Verlag für Sozialwissenschaften.

Kersting, N. (2019): Online Partizipation: Evaluation und Entwicklung - Status quo und Zukunft, In J. K. Hofmann, N. Ritzi, C. Schünemann, \& J. Wolf (Hrsg.), Politik in der digitalen Gesellschaft (S. 105-122). Bielefeld: transcript.

Lichtblau K. (2011): Die Eigenart der kultur- und sozialwissenschaftlichen Begriffsbildung. Wiesbaden: VS Verlag für Sozialwissenschaften.

Marszolek, I., Robel, Y., \& Spanka, L. (2018): Stadt, Heimat, Region. Cross-mediale Konstruktionen im Hamburg der 1950er Jahre. In A. Hepp, S. Kubitschko, \& I. Marszolek. (Hrsg.), Die mediatisierte Stadt: Kommunikative Figurationen des urbanen Zusammenlebens (S. 39-54). Wiesbaden: Springer VS.

McLeod, J., Daily, K, Guo, Z, et al. (1996): Community Integration, Local Media Use, and Democratic Processes. Communication Research, 23(2): 179-209, https://doi.org/10.1177/ 009365096023002002 . 
Nebenan.de (ohne Jahr): nebenan.de magazin. Online abrufbar unter: https://magazin.nebenan.de/ [20.07.2021].

Neuberger C. (2018): Meinungsmacht im Internet. In F. Lobigs, \& C. Neuberger (Hrsg.), Meinungsmacht im Internet und die Digitalstrategien von Medienunternehmen. Gutachten für die Kommission zur Ermittlung der Konzentration im Medienbereich (KEK) (S. 35-49). Berlin: Vistas.

Putnam, R. D. (2000): Bowling Alone: America's Declining Social Capital. Journal of Democracy, 6(1), S. 65-78.

Röper, H. (2020): Tageszeitungen 2020. Schrumpfender Markt und sinkende Vielfalt. Media Perspektiven, 2020(6), 331-352. https://www.ard-werbung.de/fileadmin/user_upload/media-per spektiven/pdf/2020/0620_Roeper_20-07-20.pdf. [20.07.2021].

Schreiber, F., Becker, A., Göppert, H., \& Schnur, O. (2017): Digital vernetzt und lokal verbunden? Nachbarschaftsplattformen als Potenzial für sozialen Zusammenhalt und Engagement - ein Werkstattbericht. FWS, 2017(4), 211-216.

Schreiber, F., \& Göppert, H. (2018): Wandel von Nachbarschaft in Zeiten digitaler Vernetzung. Explorationsstudie zur Wirkung digitaler Medien mit lokalem Bezug auf sozialen Zusammenhalt und lokale Teilhabe in Quartieren. Berlin: adelphi.

Stadt Dortmund (ohne Jahr): Smart City Dortmund. https://www.dortmund.de/de/leben _in_dortmund/umwelt/smart_city_dortmund.html [20.07.2021].

Stadt Hannover (ohne Jahr): Smart Cities. https://www.hannover.de/Service/Presse-Medien/Lan deshauptstadt-Hannover/Aktuelle-Meldungen-und-Veranstaltungen/Smart-Cities-Landes hauptstadt-beteiligt-sich-an-Initiative [20.07.2021].

Tönnies, F. (1887): Gemeinschaft und Gesellschaft. Berlin, S. [III]. In Deutsches Textarchiv, https:// www.deutschestextarchiv.de/toennies_gemeinschaft_1887/9 [20.07.2021].

Van Dijck, J., \& Poell, T. (2013): Understanding Social Media Logic. Media And Communication, 1(1), S. 2-14, https://doi.org/10.12924/mac2013.01010002.

Vetter, A. (2011): Lokale Politik als Rettungsanker der Demokratie? Aus Politik und Zeitgeschichte, 2011(7-8). http://www.bpb.de/apuz/33489/lokale-politik-als-rettungsanker-der-demokra tie [20.07.2021].

Voss, K. (2014): Internet und Partizipation: Bottom-up oder Top-down? Politische Beteiligungsmöglichkeiten im Internet. Wiesbaden: Springer VS.

Weiß, R., \& Jandura, O. (2017): Medien und gesellschaftlicher Zusammenhalt. Welche Leistungen öffentlicher Kommunikation braucht eine demokratische Gesellschaft? In O. Jandura, M. Wendelin, M. Adolf, \& J. Wimmer (Hrsg.), Zwischen Integration und Diversifikation. (S. 11-31). Wiesbaden: Springer VS.

Wellman, B., \& Hampton, K. (1999): Living Networked On and Offline. Contemporary Sociology, 28(6), S. 648-654.

Wellman, B. (2001): Physical Place and Cyber Place: The Rise of Networked Individualism. International Journal of Urban and Regional Research, 25(2), 227-252.

Wimmer, J. (2015): Überblicksartikel: Politik und Partizipation. In A. Hepp, F. Krotz, S. Lingenberg, \& J. Wimmer (Hrsg.), Handbuch Cultural Studies (S. 159-167). Wiesbaden: Springer VS.

Wimmer, J. (2017): Transformation durch Mediatisierung und Digitalisierung? Survey-Ergebnisse zum Stellenwert sozialer Netzwerkseiten und kommunikativer Orientierung für politisches Engagement. In U. Göttlich, L. Heinz, \& M. R. Herbers (Hrsg.), Ko-Orientierung in der Medienrezeption: Praktiken der Second Screen-Nutzung (S. 245-265). Wiesbaden: Springer VS. 
Anhang: Übersicht über die Variablen im Kodierbuch

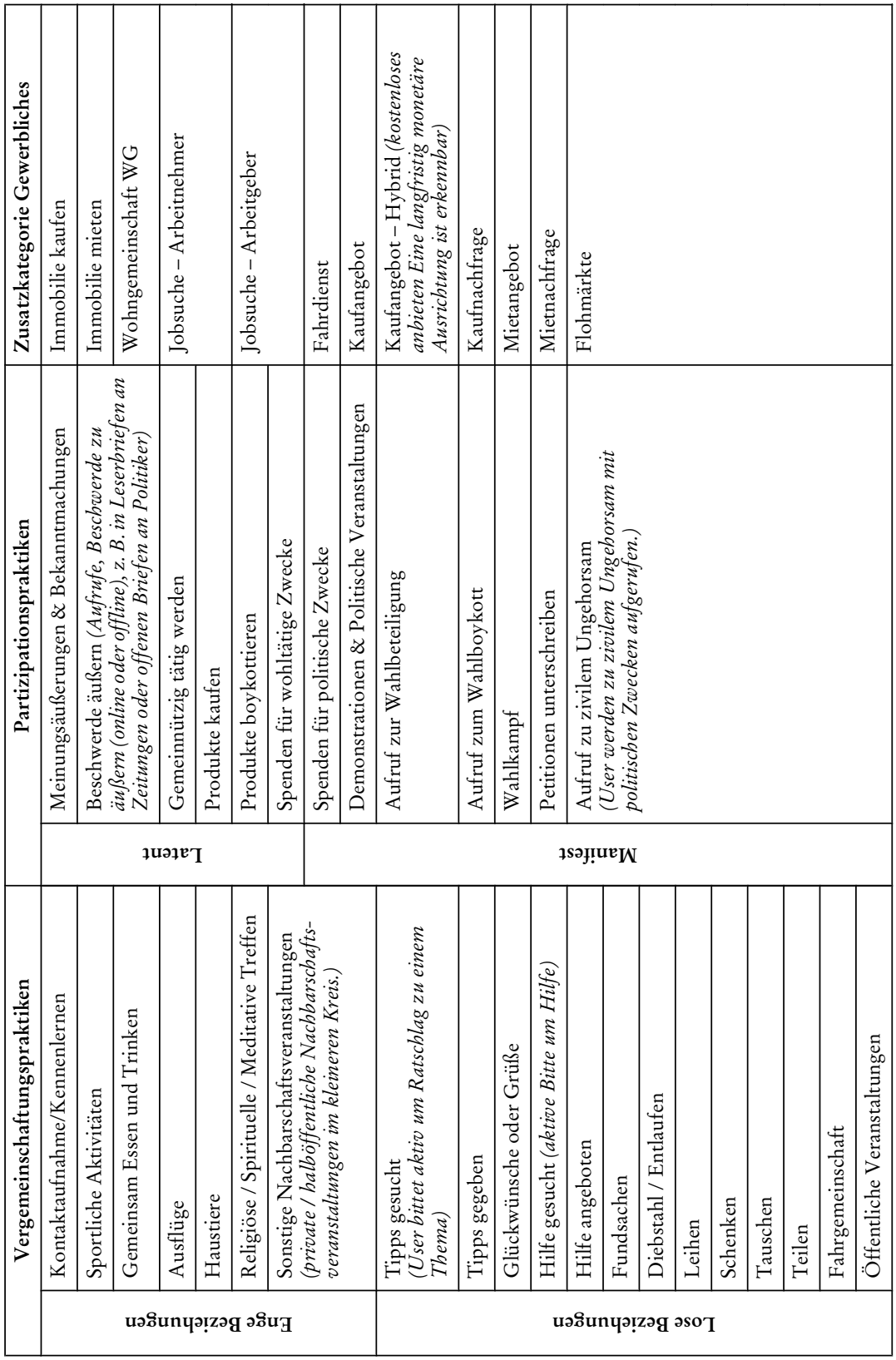

